\title{
Retinal detachment following cataract phacoemulsification-a review of the literature
}

\author{
M. Hamza Qureshi ${ }^{1,2} \cdot$ David H. W. Steel ${ }^{1,3}$
}

Received: 1 April 2019 / Revised: 25 June 2019 / Accepted: 11 July 2019 / Published online: 1 October 2019

(c) The Author(s), under exclusive licence to The Royal College of Ophthalmologists 2019

\begin{abstract}
A link between cataract surgery and rhegmatogenous retinal detachment (RRD) has long been considered. Indeed, pseudophakic retinal detachment (PPRD) forms a substantial and increasing proportion of RRD. We reviewed the literature to answer the following questions: what is the incidence of PPRD in eyes following phacoemulsification cataract surgery and how does its risk change over time following surgery? We also sought to assess how the risk is modified by intraoperative factors (operative complications, surgeon grade, subsequent laser capsulotomy), intrinsic eye-related factors (laterality, myopia, previous RRD, previous trauma, previous PVD) and patient factors (sex, age, ethnicity, affluence, systemic comorbidities). Secondarily we asked how the incidence of PPRD after phacoemulsification compares with the RRD incidence in the general population and how identified risk factors contribute to the pathophysiology of PPRD. A search of the Medline and Ovid databases was conducted for relevant publications from 1990 onwards using defined search terms with pre planned inclusion and exclusion criteria. The 10year PPRD incidence after phacoemulsification was identified as being between 0.36 and $2.9 \%$. This decreases over time to $0.1-0.2 \%$ annually but remains above the general population. The PPRD risk is further elevated by (in order of decreasing effect) intraoperative vitreous loss, increasing axial length, younger age, male sex and trainee operating surgeons. The PPRD risk after phacoemulsification is approximately ten times the general population's RRD risk. This risk is modified by the interplay of a hierarchy of risk factors, of which intraoperative vitreous loss, myopia, age and sex have the biggest effect.
\end{abstract}

\section{Introduction}

Rhegmatogenous retinal detachment (RRD) refers to the separation of the neurosensory retina from the underlying retinal pigment epithelium related to a break in the retina. RRD following cataract surgery (pseudophakic retinal detachment, or PPRD) forms a substantial and increasing proportion of RRD [1], with estimates varying from $21.6 \%$ [2] to $37.2 \%$ [3] of RRDs in the developed world being PPRDs. Cataract extraction is one of the most commonly performed surgical procedures globally with $\sim 330,000$

David H. W. Steel

David.steel@ncl.ac.uk

1 Institute of Genetic Medicine, Newcastle University, Newcastle Upon Tyne, UK

2 Royal Victoria Infirmary, Queen Victoria Road, Newcastle Upon Tyne, UK

3 Sunderland Eye Infirmary, Queen Alexandra Road, Sunderland, UK cataracts removed each year in England alone [4]. This figure is likely to increase further with an ageing population. It is important to understand the relationship between cataract extraction and PPRD as it is a serious, visionthreatening event with approximately half of all patients not recovering better than driving vision (visual acuity of approximately $6 / 12$ or better) [5].

The risk of PPRD varies with the method of cataract extraction. Conventional extracapsular cataract extraction (ECCE) carries a lower risk of PPRD by approximately one third compared with its predecessor, intracapsular cataract extraction (ICCE) [6]. ECCE has in turn been replaced almost entirely by phacoemulsification in the developed world, starting in the early 1990s [7, 8]. The early literature on phacoemulsification suggested an intermediate PPRD risk, between that of ICCE and ECCE [6]. However, these estimates may not reflect the true PPRD risk given refinements and greater familiarity and training in phacoemulsification since its early popularisation. This seems to be borne out by long-term studies reporting declining overall PPRD rates [9] and more recent studies finding equivalent [10] or lower [11] rates 
of PPRD after phacoemulsification as compared with ECCE.

The risk of PPRD after phacoemulsification is estimated variously at between $0.036 \%$ [12] and $0.656 \%$ [13] at 12 months. In comparison the incidence of primary RRD in the general population is between $0.0104 \%$ [14] and $0.0207 \%$ [3], implying that phacoemulsification increases risk of RRD by at least a factor of 1.7. This risk of PPRD is proposed to be further altered by a variety of demographic and intraoperative factors including age, sex, myopia and intraoperative complications etc.

Quantifying the risk of PPRD and the effect of these additional risk factors is important for clinicians and patients to make an informed decision before proceeding with cataract extraction. Previous reviews of the literature cannot directly answer this question as they date back to before the near-universal spread of phacoemulsification as the main surgical technique or make no distinction between phacoemulsification and other techniques (including refractive lens exchange which may confound results due to a predominantly young, myopic population with consequently higher native $\mathrm{RRD}$ risk).

In this review we attempt to draw together the findings of studies available from 1990 onwards examining the effect of phacoemulsification cataract surgery on the risk of retinal detachment and additional factors that modify this risk.

\section{Baseline risk of RRD in the general population}

The incidence of RRD in the general population is between 0.01 and $0.02 \%$ and is associated with greater socioeconomic prosperity and male sex (men are at approximately double risk). Right eyes are affected more often than left and the peak incidence of RRD is around the sixth decade of life [15]. RRD risk varies with ethnicity; Caucasians are estimated to be at tenfold higher risk than African populations [16]. Asian populations have younger age of onset $[17,18]$ but incidence varies; East Asians are at similar risk to Caucasians [14] whereas South Asians have lower risk of RRD, estimated to be threefold less than Caucasians [19, 20]. Myopia, fellow eye RRD and proliferative diabetic retinopathy, and fellow eye RRD are significantly associated with higher RRD risk [21, 22]. Nd:YAG laser capsulotomy has previously been considered a risk factor for RRD - this has more recently been challenged [23].

In $80-90 \%$ of RRDs the precipitating event is a retinal break associated with a posterior vitreous detachment (PVD) [24]. PVD is a natural, age-related result of progressive vitreous liquefaction and increases rapidly around age 60-70 years. It has been estimated that, in between 8 and $15 \%$ of people affected, this is associated with retinal break formation [25, 26]. It has also been suggested that established and complete PVD without RRD at presentation is protective against RRD when cataract surgery is performed [22, 27].

\section{Methodology and search strategy}

We conducted a search of the Medline and Ovid databases for all publications from 1990 using the search terms: (retinal detachment) AND (pseudophak* OR phacoemulsification OR (Cataract AND (surgery OR operation OR extraction OR removal))).

This yielded 2634 results which were supplemented by manual searches primarily using additional references from key articles. Inclusion criteria included: prospective or retrospective studies of RRD incidence in a predominantly postphacoemulsification population and English language. Papers which met our criteria were analysed for PPRD incidence overall and by risk factor. Any papers whose eligibility was not clear from the abstract were retrieved and read to ascertain whether they merited inclusion. Exclusion criteria were: population size of $<1000$ pseudophakic eyes, inclusion of phacoemulsification performed pre-1990, inclusion of refractive (clear) lens exchange phacoemulsification, populations with $>40 \%$ ICCE or ECCE, solely paediatric population and incomplete data.

A summary of the findings of the 16 papers that met these criteria is provided in Table 1 and discussed below. In summary, the population sizes ranged from 1793 to 2,680,167 with an estimated total of 3,211,671 pseudophakic eyes. Four papers were by the same Taiwanese group (Sheu et al.) following a fixed population over multiple years of follow-up $[10,28-30]$. In addition to these four, a fifth study by a different group was also based in Taiwan [31], a sixth in Singapore [32] and a seventh in New Zealand [33]. All other studies investigated phacoemulsification in European centres. Minimum study follow-up durations from the time of cataract surgery ranged from no defined minimum duration [11, 31] (i.e. PPRD incidence calculated over the same time periods as phacoemulsification) to 10 years post-phacoemulsification [33]. Three studies retrospectively calculated the frequency of individual risk factors present in PPRD eyes compared with the frequency in unaffected pseudophakic eyes [32, 34, 35]. All other studies stratified the pseudophakic population by presence or absence of risk factors and prospectively calculated the incidence of PPRD.

\section{Overall incidence in pseudophakic population}

The reported incidence of PPRD varied considerably between studies and also with the length of follow-up. 


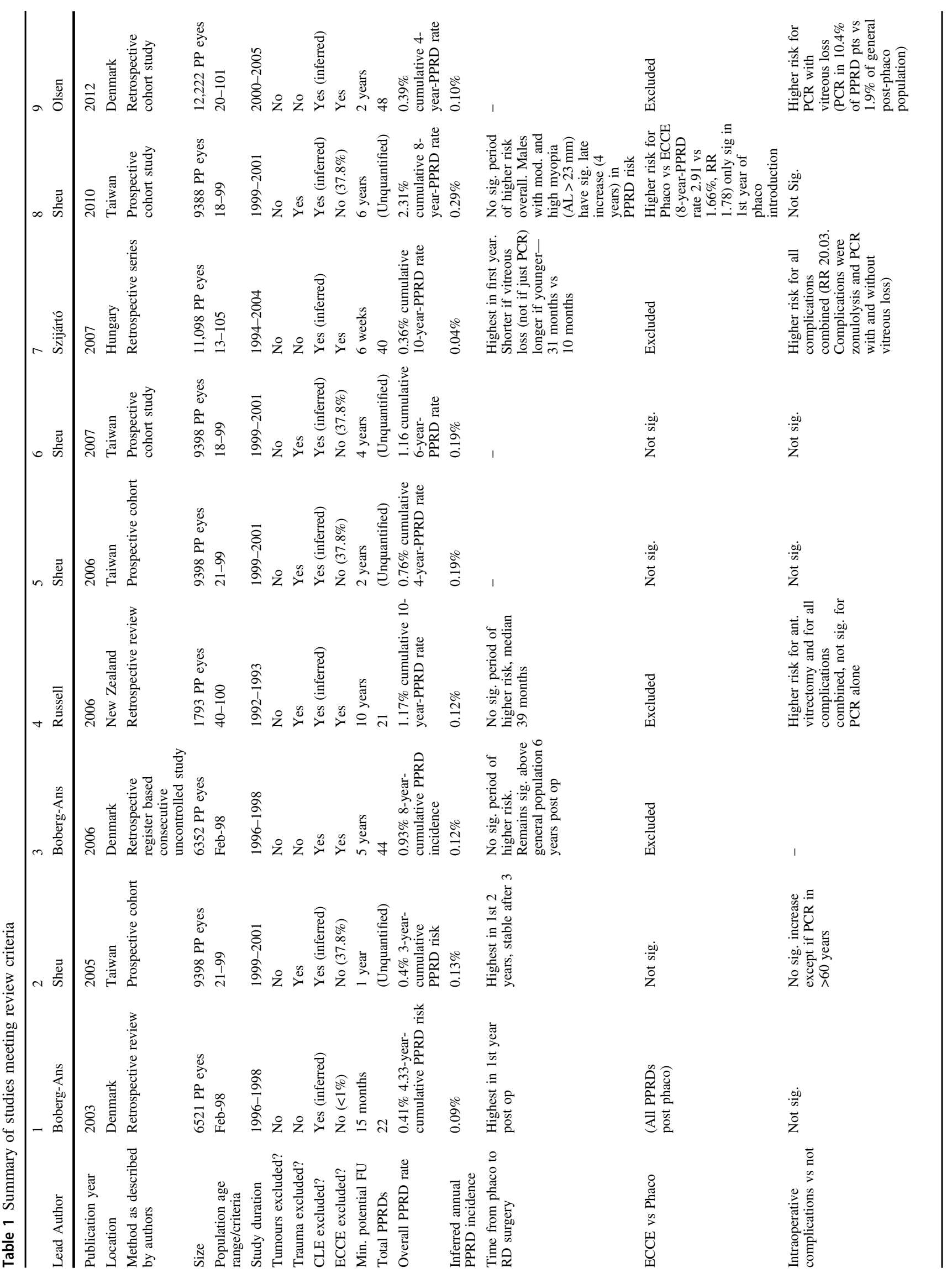




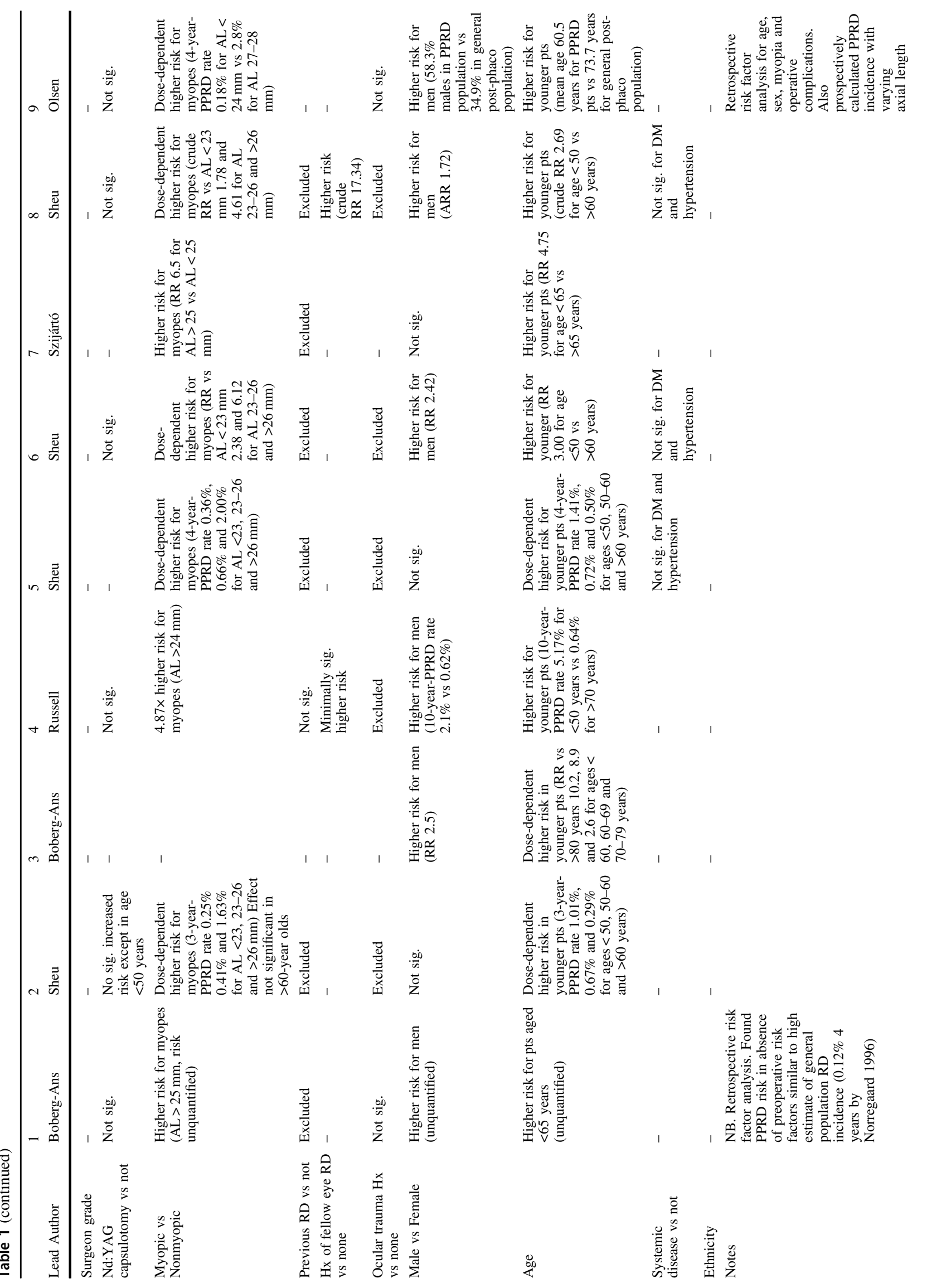




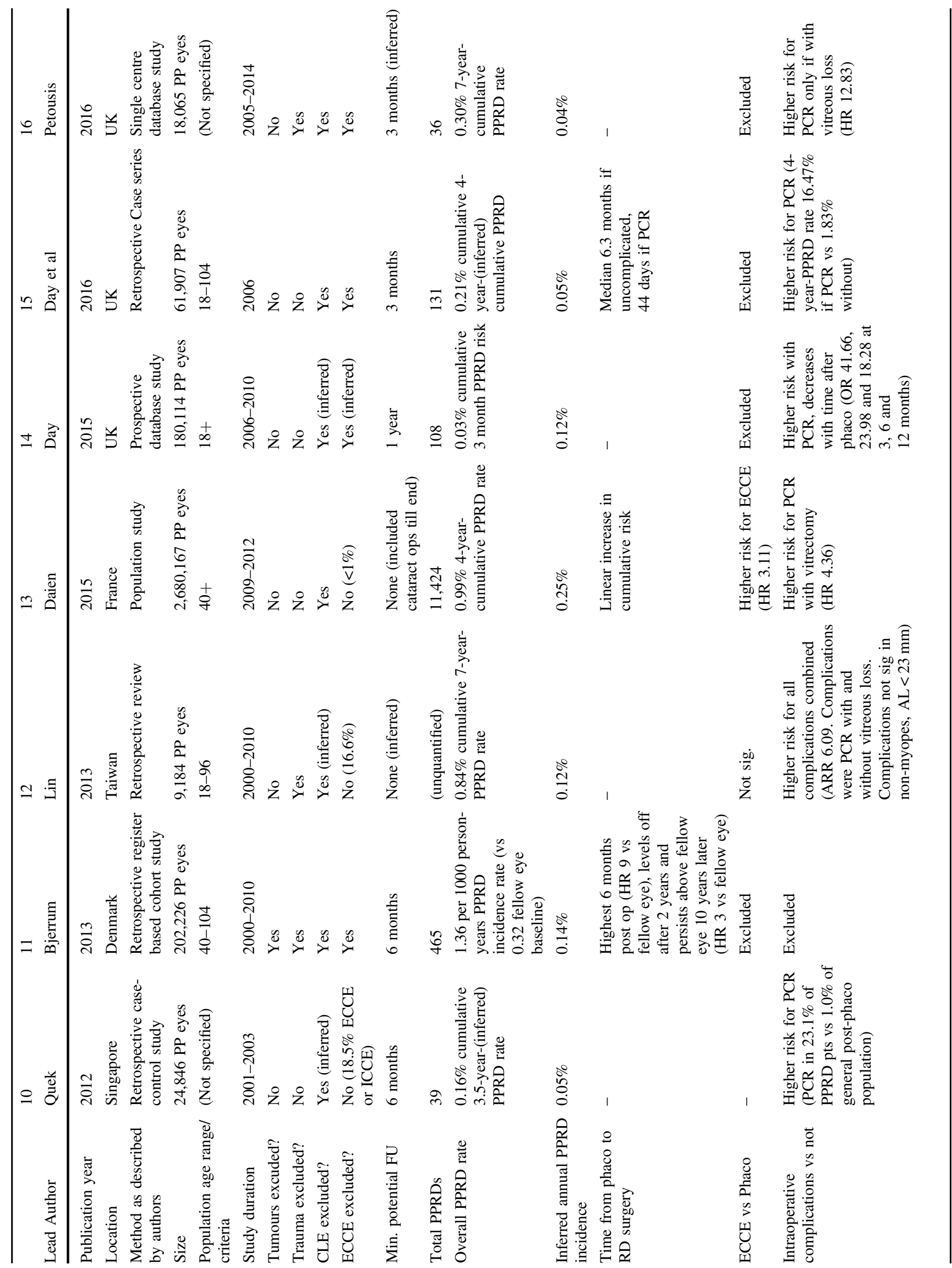




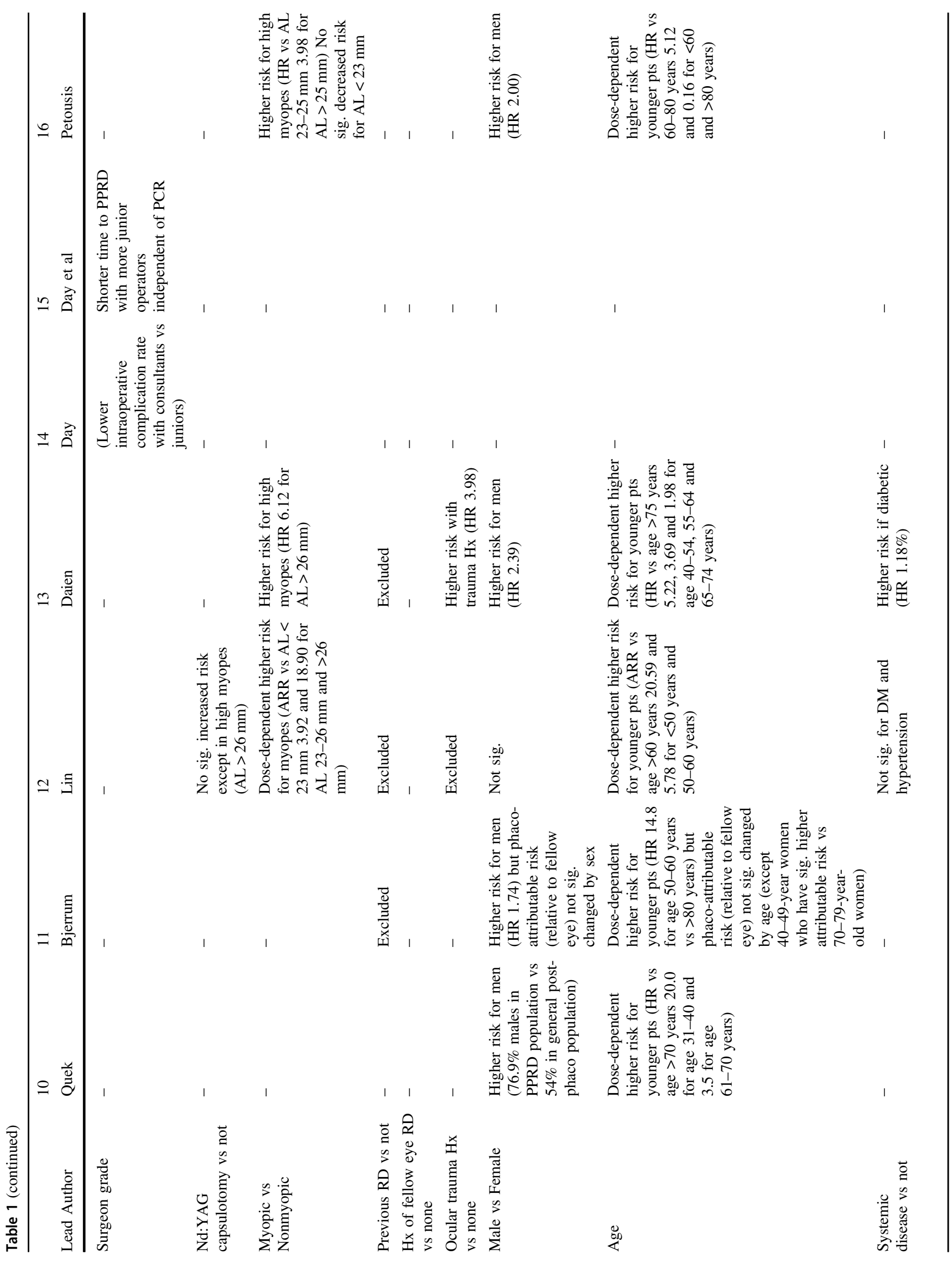




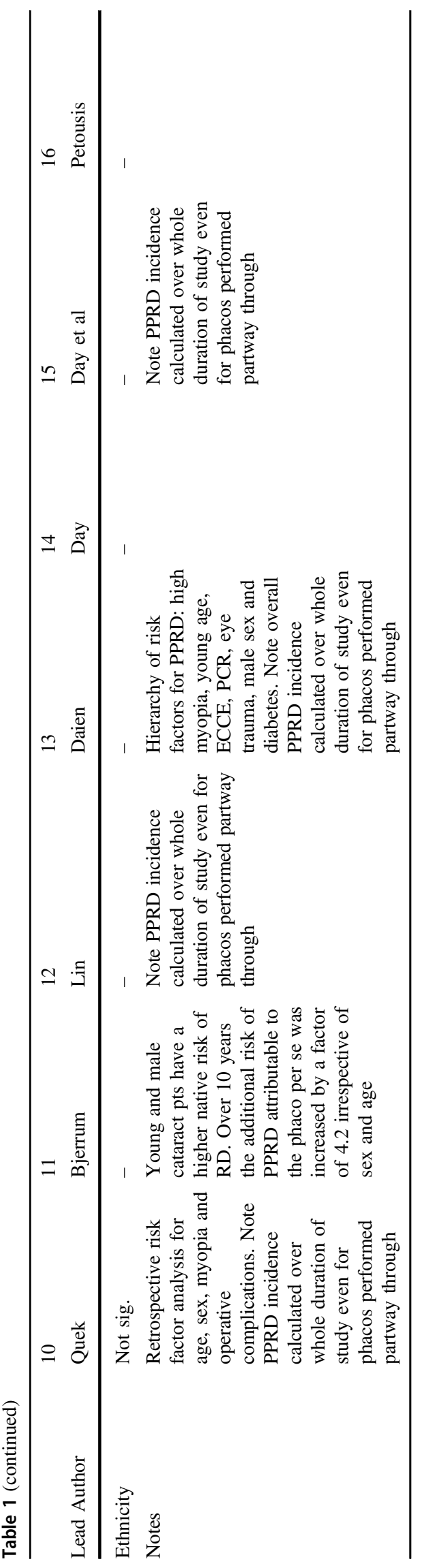

The lowest reported cumulative incidence of PPRD was $0.36 \%$ over 10 years [12], equating to an annual incidence of $0.036 \%$ (by Szijártó et al. in Hungary) while Sheu et al. reported $2.31 \%$ over 8 years in their final published follow-up [10], equating to $0.289 \%$ per annum which is the highest value in our review. In general however, studies with longer follow-up report a greater cumulative incidence of PPRD with an approximately linear relationship corresponding to an incidence of $0.1-0.2 \%$ for each addition year after phacoemulsification (see Fig. 1). This is supported by evidence that the risk of RRD in pseudophakic eyes remains significantly elevated for over a decade after the operation [36].

\section{PPRD risk over time following phacoemulsification}

Nine studies investigated how the PPRD incidence evolves with time from phacoemulsification. Three found no period of increased incidence [11, 33, 37] while one found a late increase in incidence after 4 years for myopic males only [10]. A further three reported a higher PPRD incidence in the first 6-24 months postphacoemulsification $[28,34,38]$. An eighth study found a significantly shorter median time from phacoemulsification to PPRD (44 days compared with 6.3 months) if the eye suffered intraoperative posterior capsule rupture (PCR) or if the operator was a trainee surgeon independently of whether PCR occurred (figures not given) [39]. Notwithstanding the short follow-up in this study (minimum 3 months) the implication is that the early post-operative period carries higher risk of PPRD. Moreover this finding is corroborated by a ninth study which found the first year after phacoemulsification to have the greatest risk of PPRD and that the median time to PPRD was shortened from 31 months to 10 months in eyes which suffered PCR with vitreous loss [12].

The five studies reporting increased PRPD risk in the early post-operative period do not necessarily negate an additional long-term increase in PPRD risk. Indeed Bjerrum et al. note that the while the highest PPRD risk is during the first 6 months (Hazard Ratio, HR of 9 relative to the un-operated fellow eye) and then decreases, the PPRD risk plateaus at 2 years (HR 3) and remains higher than the fellow eye for a decade [38].

While the case for an early increased PPRD risk is compelling given the link with intraoperative complications, it may also in part reflect greater surveillance in the early post-operative period, especially in complicated cases, and increasing patients lost to follow-up with time. 


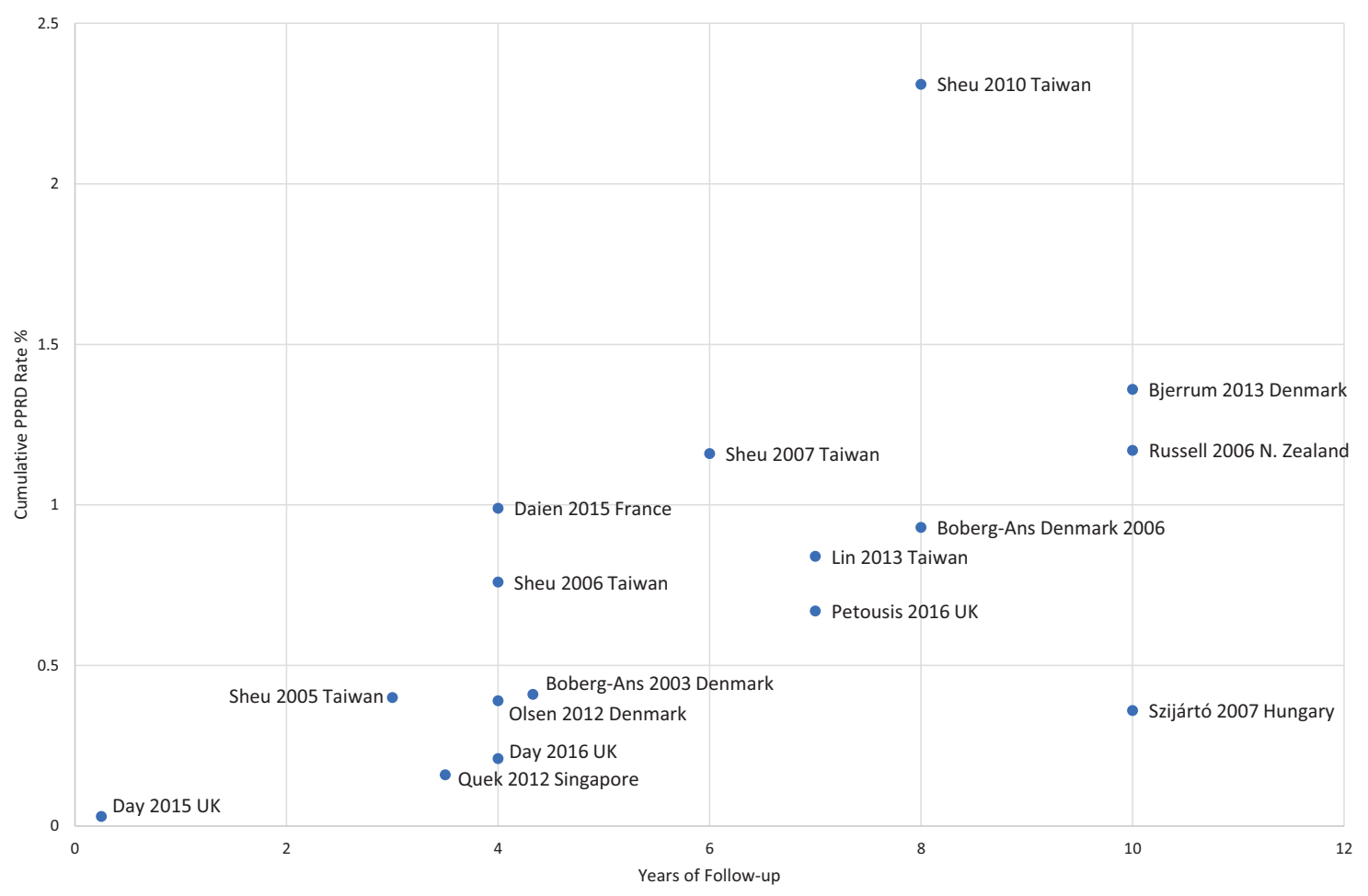

Fig. 1 Cumulative PPRD incidence as reported by the 16 studies included in this review

\section{PPRD risk after phacoemulsification in comparison with ECCE}

As earlier estimates may have overestimated the PPRD risk after phacoemulsification due to the technique's unfamiliarity, we selected studies in which phacoemulsification was the predominant cataract extraction method. Nevertheless, six studies which included some ECCE cases compared the PPRD incidence in ECCE and phacoemulsification cases.

Daien et al. found a higher PPRD incidence after ECCE cases (HR 3.11) [11]. All other studies reported no significant difference [28-31] with the exception of Sheu et al. who in their final follow-up found phacoemulsification was associated with a higher PPRD incidence (Relative Risk, $\mathrm{RR}$ of 1.78). Interestingly in their previous follow-up papers (2005-2007) this difference did not reach significance in the overall population. Moreover, they note that in their final study that the increased risk after phacoemulsification was attributable to cases from the first year of their operative period (c.1999). This period corresponded to a changeover from ECCE to phacoemulsification and the two techniques had no significant difference in PPRD incidence when only considering cases from the following year [10].

Unifying the results of these studies it would appear that phacoemulsification is as safe or safer than ECCE once the technique is familiar. For centres transitioning from ECCE to phacoemulsification, the newer technique may well have a greater PPRD risk and previous studies which found a higher PPRD risk after phacoemulsification than ECCE should be viewed as overestimates in light of this. Conversely, we note (as did Daien et al.), that current estimates of higher PPRD risk after ECCE than phacoemulsification may be confounded by the fact that in centres where phacoemulsification is the dominant technique, ECCE is now reserved for more challenging cases such as cataracts with denser crystalline lenses not amenable to phacoemulsification.

\section{Intraoperative complications}

All groups who examined the effect of intraoperative complications (vitreous loss or PCR), found a significant association with increased PPRD except Sheu et al. and BobergAns et al. in their first study [34]. The estimated increase in risk varied from approximately fourfold at 4 years [11] to as high as 42 times higher risk of retinal detachment surgery within 3 months following PCR [4]. The latter figure is from Day et al. who found this higher initial PPRD risk following PCR decreased over time (OR 23.98 and 18.28 at 6 and 12 months, respectively). As previously mentioned, the 
same group also found in a different study that PCR was associated with a shorter time to PPRD [39].

In the only other study to examine the effect of intraoperative complications on the time from phacoemulsification to PPRD, Szijártó et al. likewise found PCR was associated with shorter time to PPRD but only if accompanied by vitreous loss [12]. This point is also borne out by the observation that among the nine studies which found higher incidence of PPRD in eyes suffering intraoperative complications, five considered intraoperative complications collectively (i.e. without distinguishing the presence or absence of vitreous loss) [4, 12, 31, 32, 39] while four specified PCR with vitreous loss as being associated with higher PPRD risk [11, 33, 35, 40]. Of these, Russell et al. and Petousis et al. specifically compared the PPRD risk in eyes suffering PCR with and without vitreous loss and found increased PPRD only in cases of PCR with vitreous loss, with no significant increase in PPRD risk in eyes suffering PCR without vitreous loss. The implication of this would be that intraoperative complications per se are not associated with higher PPRD risk, rather vitreous loss is. This may be to do with the pathophysiology of PPRD, as explored below, but has significance in terms of detection, management and training of PCR intraoperatively.

\section{Surgeon grade}

One study meeting our inclusion criteria examined the effect of surgeon grade on PPRD by classifying operators into trainee, independent non-consultant and consultant surgeons. They found a shorter time from phacoemulsification to RRD in eyes operated on by more junior surgeons [39]. The authors did not comment on whether the overall rate was higher after cataract extraction by junior surgeons but in light of this finding this is likely to be the case at least in the immediate post-operative period. Given the short follow-up time of this study it is unclear whether this only reflects a higher early incidence and whether over longer post-operative follow-up the PPRD incidence is significantly different if operated on by consultants.

Intraoperative complications are more likely at the hands of a trainee surgeon [4] and surgeon grade is a variable in the current PCR risk calculator [41]. However, this study finds the lower surgeon grade increases PPRD risk independently of PCR. If this is the case it could be hypothesised that the increased risk also relates to greater anterior chamber fluctuations, more prolonged surgery and infusion volumes into the eye.

More generally, familiarity of surgical teams with phacoemulsification decreases PPRD risk as demonstrated by Sheu et al. who showed that a transition from ECCE to phacoemulsification was marked by initially higher PPRD incidence following phacoemulsification. This became nonsignificant 1 year after transition away from ECCE as the predominant cataract extraction technique [10].

\section{Nd:YAG capsulotomy}

Nd:YAG capsulotomy has previously been associated with increased post-cataract extraction RRD risk [42, 43] but all seven studies meeting our criteria found no significant link with increased PPRD overall in their post-phacoemulsification population. However in a sub-group analysis, Lin et al. found that high myopes had an increased PPRD risk if they underwent Nd:YAG capsulotomy [31]. Similarly Sheu et al. in their first follow-up found an association in their sub-group of patients aged under 50 years but none in their overall pseudophakic population [28]. Unfortunately, although Sheu et al. subsequent follow-up studies confirmed that capsulotomy is not significant overall, they did not revisit whether this remained significant in younger eyes over a longer period of follow-up.

Unlike studies focusing only on post-capsulotomy PPRD risk (none of which met our inclusion criteria), the studies in our review were all primarily concerned with postphacoemulsification risk with Nd:YAG capsulotomy considered secondarily. Because of this, their results may be confounded by follow-up times that were calculated from phacoemulsification rather than from capsulotomy. None of the included studies gave data on time lags between phacoemulsification and capsulotomy, introducing an uncontrolled variable. Moreover, given that this may have occurred as much as 2 years after phacoemulsification this shortens the time during which post-capsulotomy PPRDs can occur and would be expected to underestimate the effect of laser capsulotomy when incidence is calculated from the time of phacoemulsification.

This uncertain significance of laser capsulotomy on PPRD risk in our selected studies is in keeping with more recent studies [44], including a review of the literature which suggests that there is no convincing association between Nd:YAG capsulotomy and PPRD [23], though, like Lin et al., the authors note that a significant association may exist for myopic eyes undergoing capsulotomy.

These observations may be reconciled with findings to the contrary, especially by older studies, in light of a study by Olsen and Olson which considered 1099 ECCE and 1418 phacoemulsification cases, with approximately one third of each group going on to have laser capsulotomy. They found that whilst Nd:YAG capsulotomy was significantly associated with PPRD after ECCE, it had little impact on PPRD risk in the context of phacoemulsification [45], perhaps relating to consistent intracapsular IOL 
fixation and more assured 'separation' of the anterior and posterior segments.

\section{Axial length and myopia}

As with phakic RRD, myopia was strongly associated with increased PPRD risk in all 11 studies that considered it. All studies used axial length rather than refractive error and the definition of myopia varied between the various groups from $>23 \mathrm{~mm}$ to $>25 \mathrm{~mm}$. Estimates varied between 2.74 [40] and 18.90 [31] times increased risk compared with non-myopes. Sheu et al. found female sex [30] and older age [28] were protective against the increased PPRD risk from myopia.

Six studies grouped their patients by axial length and demonstrated a dose-dependent relationship between axial length and PPRD risk. Lin et al, who found the most pronounced effect, found eyes with axial length $23-26 \mathrm{~mm}$ had a non-significant adjusted relative risk of 3.92 compared with eyes with axial length $<23 \mathrm{~mm}$ while eyes with axial length $>26 \mathrm{~mm}$ had a significantly increased relative risk of 18.90. [31]. Importantly they also found a significant, dosedependent protective effect of being non-myopic against the effects of young age and intraoperative complications (see Table 2).

\section{Previous RRD in operated eye}

Previous RRD is a strong risk factor for phakic redetachment in the same eye. On this basis it might be expected that after phacoemulsification such eyes would likewise show a higher PPRD incidence. Forsell et al. recently demonstrated a low rate of PPRD in a small group of patients with previous RRD in the operated eye [46]. Unfortunately, this study did not meet our criteria and the majority of those that did excluded eyes with previous RRD. The only study in our review to investigate the effect of previous RRD on PPRD risk in the same eye found no significant difference, unsurprisingly given the small numbers concerned ( 2 PPRDs in a population of 11 eyes with RRD preceding phacoemulsification) [33].

\section{Previous RRD in fellow eye}

A previous RRD in the fellow eye is known to be a strong risk factor for phakic RRD in the contralateral eye. However only two studies examined the significance of fellow eye RRD on PPRD on the contralateral side. Russell et al. found a minimally significant increased risk if the fellow eye had suffered a RRD [33], while Sheu et al. found such
Table 2 Reproduced from Lin et al. 2013

\begin{tabular}{|c|c|c|c|c|c|c|}
\hline \multirow[t]{2}{*}{ Variables } & \multicolumn{2}{|l|}{$\begin{array}{l}\mathrm{AL}<23 \mathrm{~mm} \\
(N=3240)\end{array}$} & \multicolumn{2}{|l|}{$\begin{array}{l}23 \mathrm{~mm} \leq \mathrm{AL}<26 \\
\mathrm{~mm}(N=5518)\end{array}$} & \multicolumn{2}{|l|}{$\begin{array}{l}\mathrm{AL} \geq 26 \mathrm{~mm} \\
(N=426)\end{array}$} \\
\hline & $\begin{array}{l}\text { Adjusted relative } \\
\text { risk }(95 \% \mathrm{CI})\end{array}$ & $P$ value & $\begin{array}{l}\text { Adjusted relative } \\
\text { risk }(95 \% \mathrm{CI})\end{array}$ & $P$ value & $\begin{array}{l}\text { Adjusted relative } \\
\text { risk }(95 \% \mathrm{CI})\end{array}$ & $P$ Value \\
\hline \multicolumn{7}{|l|}{ Age } \\
\hline$>60$ & 1 & & $1.75(0.18-16.81)$ & 0.63 & $10.99(0.69-175.75)$ & 0.09 \\
\hline $\begin{array}{c}50< \\
\text { age } \leq 60\end{array}$ & $\begin{array}{l}0.04(0-7.182 \mathrm{E}+ \\
11)\end{array}$ & 0.84 & $\begin{array}{l}13.78 \\
(1.25-151.99)\end{array}$ & $0.03 *$ & $34.51(3.09-385.58)$ & $0.004 *$ \\
\hline$\leq 50$ & $\begin{array}{l}0.05(0-4.359 \mathrm{E}+ \\
27)\end{array}$ & 0.93 & $\begin{array}{l}36.67 \\
(3.30-407.92)\end{array}$ & $0.003 *$ & $\begin{array}{l}151.80 \\
(15.63-1474.46)\end{array}$ & $<0.001 *$ \\
\hline \multicolumn{7}{|c|}{ Capsulotomy } \\
\hline No & 1 & & $2.81(0.33-24.30)$ & 0.35 & $7.25(0.57-92.44)$ & 0.13 \\
\hline Yes & $0.03(0-54286105)$ & 0.76 & $3.94(0.35-44.55)$ & 0.27 & $43.50(3.56-532.22)$ & $0.003^{*}$ \\
\hline \multicolumn{7}{|l|}{ Sex } \\
\hline Female & 1 & & $\begin{array}{l}0.02 \\
(0-136817.09)\end{array}$ & 0.61 & $9.61(0.74-124.63)$ & 0.08 \\
\hline Male & $\begin{array}{l}0.01 \\
(0-658818.80)\end{array}$ & 0.59 & $3.56(0.39-32.31)$ & 0.26 & $8.57(0.87-84.78)$ & 0.07 \\
\hline \multicolumn{7}{|c|}{ Intraoperative complications } \\
\hline No & 1 & & $3.44(0.41-28.89)$ & 0.26 & $19.08(1.89-192.28)$ & $0.01 *$ \\
\hline Yes & $\begin{array}{l}0.04(0-9.947 \mathrm{E}+ \\
18)\end{array}$ & 0.9 & $\begin{array}{l}14.57 \\
(0.91-232.92)\end{array}$ & $0.05^{*}$ & $\begin{array}{l}46.73 \\
(1.71-1278.01)\end{array}$ & $0.02 *$ \\
\hline
\end{tabular}

$C I$ confidence interval, $A L$ axial length

* $P$ value was considered to be significant 
eyes at much higher risk of PPRD risk (crude RR 17.34) [10]. The latter study had a much larger population size (9388 pseudophakic eyes in Sheu et al. compared with 1793 in Russell et al.) though the number of operated eyes with a history of fellow eye RRD is small in both (six in Sheu et al. and four in Russell et al.) and is more likely to account for the large difference in findings. We would also expect that the nature of the fellow eye RRD may alter the risk of PPRD differently depending on how long ago it occurred and whether the detachment was a primary rhegmatogenous RRD or secondary to trauma or an intraocular tumour. The dearth of available data addressing this is likely due to the comparatively small number of eyes in this category making further subdivision of fellow eye RRD unfeasible.

\section{Ocular trauma}

Ocular trauma is a known risk factor for RRD. The incidence of RRD following trauma is estimated at around 5\% over 6 months [47]. In our review of PPRD risk factors, many studies excluded eyes with a history of trauma. Of the ten that did not, only three examined the effect on PPRD incidence. In two retrospective studies, Boberg-Ans et al. and Olsen et al. found that eyes that had suffered PPRD had no significantly higher chance of having had a history of ocular trauma [34, 35]. Both studies were comparatively small, especially when considering their subpopulation of eyes which had previously suffered trauma (Boberg-Ans et al. found 1 eye with previous trauma out of 22 PPRDs in a total population of 6521 pseudophakic eyes and Olsen et al. found 7 eyes with previous trauma out of 48 PPRDs in a total population of 12,222 pseudophakic eyes).

In contrast, in a much larger study (203 eyes with previous trauma out of 11,424 PPRDs in a total population of 2,680,167 pseudophakic eyes) Daien et al. found eyes with a history of trauma were at significantly increased PPRD risk (HR 3.98) [11]. This finding is more in keeping with the known increased RRD risk in the general population after ocular trauma and so the findings of the earlier two studies may be the result of smaller population sizes.

It is also conceivable that the risk of PPRD in such eyes varies with the nature and severity of the trauma, how long ago it occurred, whether it disrupted the vitreous or retina and what, if any, treatment was given. Given these additional factors, ocular trauma per se may not be as helpful a variable in calculating PPRD risk as defined injuries to specific anatomical structures. Realistically however it may be difficult to recruit populations of sufficient size to be able to further subdivide eyes in such a way.

\section{Gender}

In the general population, RRD is more common in men than women, with the sex ratio estimated at between 1.5:1 [48] and 2:1 [1]. Of the 14 studies investigating the effect of sex on PPRD risk after phacoemulsification, 10 found a significantly greater incidence of PPRD in men, with the increased risk between 1.72 [10] and 3.39 [33] times that of women. Of the remaining four, two are the 2005 and 2006 studies by Sheu et al. who went on to demonstrate in their subsequent studies of the same population that with increasing follow-up men did have a higher PPRD risk than women [10, 30].

This raises the question of whether the increased PPRD incidence in men is genuinely attributable to phacoemulsification or whether over such long follow-up the increased incidence reflects the higher background RRD risk for men. Exploring this, Bjerrum et al. compared PPRD incidence in the operated eye with RRD in the un-operated fellow eye, finding that, when adjusted for the higher PPRD incidence in male un-operated fellow eyes, the attributable increase in PRPD risk of male operated eyes was not significantly more than women, i.e. phacoemulsification increases the risk of RRD uniformly irrespective of sex and the higher incidence of PPRD in males is due to a higher pre-operative risk [38].

If this is true, men are still at greater risk of PPRD than women following phacoemulsification but to understand why this is the case pre-operative risk factors for RRD are more significant. Olsen et al. suggest the answer lies in male eyes being longer than female eyes (i.e. a higher incidence of myopia in men), which is a known risk factor for RRD [35]. While this is true of the Danish population studied by Olsen et al. [49] and has been invoked to explain the male preponderance of RRD in a Scottish population [50], the global picture is more unclear. While women show higher incidence of hyperopia (Odds ratio, OR 1.28), myopia shows similar prevalence in men and women globally when adjusted for age and race. Moreover in some populations women have significantly higher rates of high myopia than men, (e.g. OR 1.61 in white Australians) [51]. It has also been suggested that posterior migration of the posterior border of the vitreous base contributes to the higher incidence of RRD in men [50, 52].

Furthermore, the higher PPRD risk in men seems to be independent of the effect of age, axial length and previous ocular trauma. In a population excluding eyes with a history of trauma, Sheu et al. found female sex mitigates the additional risk from young age and myopia, strengthening the case for a different explanation which they suggest may be a greater incidence of unreported ocular trauma in males than females [30]. 


\section{Age}

All papers examining age as a risk factor found young age significantly associated with PPRD, contrary to the wider population's increased risk or RRD with age up to around 60 . Those studies which grouped their study population into age brackets all found a dose-dependent relationship between increasing age and lower PPRD risk. The most modest increase is reported by Sheu et al. who, in their final follow-up study, found a relative risk of 2.69 for patients aged under 50 compared with those aged over 60 years [10]. The largest age-dependent risk modification is reported by Petousis et al. who found those aged under 60 at $\sim 40$-fold greater risk of PPRD than those aged over 80 years [40]. This lower PPRD risk in older patients appears to be independent of sex and also mitigates the additional PPRD risk in myopic eyes [28].

Bjerrum et al. note that above the age of 50 PPRD risk drops exponentially by just over half for each decade [38], a relationship which also approximates to the results of several other studies in this review, although due to differences in age bracketing this is difficult to quantify. This observation is also in line with an older estimate of change in PPRD risk following ECCE by a Swedish group who found PPRD risk changed by a factor of 0.94 for each additional year of age, which when raised to the tenth power corresponds to a factor of 0.54 over a decade [53].

However, Bjerrum et al. found this increased risk also applied to RRD in fellow un-operated eyes, i.e. phacoemulsification increases the risk of RRD uniformly irrespective of age. This would suggest that higher incidence of PPRD in younger patients is due to a higher pre-operative risk [38]. Given younger eyes in the general population have a lower native RRD risk, the higher risk in these patients may presumably be attributed to pathological processes which predispose both to early cataracts and easily detached retinas.

\section{Systemic diseases}

Of the studies considering the impact of systemic diseases (primarily hypertension and diabetes), all found no significant effect [10, 29-31] other than Daien et al. who found an increased PPRD risk in diabetics (HR 1.18) [11]. If so, this may be the result of traction on the retina from proliferative diabetic retinopathy which is a known risk factor for RRD in the general population and was implicated in a British study which found a rise in RRD attributable to an increased prevalence of diabetes [54].

\section{Ethnicity}

Comparisons of RRD incidence in the general population have found differences depending on ethnicity with Caucasian, Asian and African populations associated respectively with higher, intermediate and lower RRD risk. Quek et al's study of a Singaporean population is the only one in our review to examine the risk of PPRD as affected by ethnicity, finding no significant effect [32]. This study is limited by its short follow-up duration (minimum 6 months) and consequently low number of PPRDs (total 39) making underestimation of risk more likely. The authors also did not specify which ethnicities they compared in their population. This is especially important as the literature on the effect of race on phakic RRD incidence suggests a tendency among East Asian authors to compare within Asian subpopulations (Chinese, Indian, Malay etc.) whereas Western authors tend to compare more broadly between Caucasian, Asian or African populations. This methodological difference may significantly alter whether or not a particular study finds differences in RRD or PPRD incidence

\section{Other risk factors}

In the general population right eyes are at greater risk of RRD than left eyes. No studies that met our criteria examined the effect of laterality on PPRD risk, although it has been postulated in phakic eyes that it may be related to ocular dominance and myopia. Likewise, while some studies have linked affluence with increased RRD risk, there is currently no literature investigating this as a risk factor for PPRD specifically. Similarly, although PVD is known to be associated with RRD, we found no studies meeting our criteria which considered the effect of PVD on PPRD incidence.

\section{Phacoemulsification-attributable risk}

Bjerrum et al. highlighted a largely neglected consideration which is that the increased rate of RRD in the pseudophakic population is not necessarily attributable to cataract extraction. Cataract-prone eyes may have an increased native risk of RRD and a small study of 64 patients has previously demonstrated increased risk of RRD in the unoperated fellow eyes of PPRD patients [55]. As such the most appropriate control, rather than being the general population, is the fellow eyes of the same patients. With this in mind they still find a significant attributable risk to phacoemulsification (1.36 PPRDs per 1000 person-years in 
the operated eye compared with 0.32 in the fellow eye). Interestingly however, while they found young age and male sex significantly associated with PPRD, this became insignificant when compared with the comparably increased fellow eye RRD risk in these patients. The implication of this would be that while young males with cataracts are at higher risk of RRD, the phacoemulsification procedure increases the risk uniformly (by a factor of 4.2 in this study) irrespective of age or sex.

As this study remains the only one to present data on fellow eye RRD incidence and only investigated age and sex, we are unable to comment definitively on how this would modify the attributable risk of PPRD as calculated by other groups and how this would vary with other risk factors, but we would expect it to be lower than previous calculations comparing with the wider, cataract-free population. Nevertheless, even with this analysis, the additional PPRD risk following phacoemulsification will precipitate more PPRDs in populations whose native RRD risk is high.

This methodological approach is hampered however by the typically short duration between phacoemulsification of one eye and its fellow. Furthermore, if a cataractous eye has a higher native risk of RRD, the fellow eye may have a reduced native RRD risk if it does not develop a cataract till later in life making it no better a control than the general population-Bjerrum et al. note however that their study period was marked by many patients who developed cataracts simultaneously but had long waiting times between cataract extractions which may mitigate this to some degree. Perhaps most conspicuously, this study excluded eyes that suffered intraoperative complications, a risk factor unambiguously related to the phacoemulsification procedure and not shared with the fellow eye.

Finally, this approach underemphasises the potential effect of a small additional risk on eyes with an already high baseline RRD risk. From a patient perspective, overall PPRD risk is the key concern-even if the proportion of this risk attributable to phacoemulsification is small, this is more likely to precipitate $\mathrm{RRD}$ in populations at higher baseline risk.

\section{Pathophysiology of PPRD}

In an effort to establish a causative link between cataract extraction and PPRD, Mahroo et al. analysed the clinical features of phakic RRDs and PPRDs in 500 eyes and found differing patterns in position and size of breaks between the two groups [56]. In comparison with phakic RRDs, PPRDs presented less frequently with vitreous haemorrhage and more frequently with multiple breaks, breaks smaller than 0.5 -disc diameter and with breaks in the inferonasal quadrant and at the more surgically challenging 5 o'clock to 7 o'clock position. To further dissect whether the difference in pathophysiology of phakic RRDs and PPRDS is purely due to phacoemulsification or the cataractous eye itself, the authors further compared between phakic eyes with cataract and pseudophakic eyes, finding a similar pattern of differing clinical features between the RRDs of both groups as they did between pseudophakic eyes compared with all phakic eyes. If replicable, this study's results would strongly support the phacoemulsification procedure as contributing a distinct additional risk.

It has been suggested that the mechanism of PPRD involves vitreous changes during and after phacoemulsification [57]. This draws on the fact that RRD in phakic eyes is typically preceded by PVD [24] although only a small proportion of PVDs are associated with RRD. The agerelated liquefaction of the vitreous that naturally results in PVD progresses most rapidly in the sixth decade of life, explaining the peak incidence of RRD. The increased RRD risk with PVD is in the acute setting, after which established (chronic) PVD is believed to be protective against subsequent RRD [22, 27].

A similar process may occur as a result of acute vitreous traction caused by movement of the lens capsule during phacoemulsification. This would also explain why PCR and specifically vitreous loss so dramatically increases the risk of PPRD in the studies we reviewed, and why this increased risk seems to become less marked over time. This mechanism would also explain the higher incidence of PPRD after ICCE.

Changes in vitreous tractional forces may also underpin the long-term increased rate of PPRD beyond the early postoperative period. The protuberance of the posterior surface of the native lens is thought to reduce vitreous traction on the peripheral retina [58]. This protective effect may be reduced in pseudophakia and lost in aphakia.

Changes in the composition of the vitreous may also be implicated. A post-mortem study of three pseudophakic and seven phakic eyes found changes in the protein composition and structure of the vitreous humour after phacoemulsification that were not present in the phakic eyes which included two un-operated fellow eyes. The authors noted in particular the presence of crystallins (which are absent in phakic eyes) in the anterior vitreous and lower viscosity in the anterior than the posterior vitreous (which represents a reversal of the viscosity gradient in phakic eyes). The authors conclude that the vitreous' normal protein processing and clearance mechanisms are altered in pseudophakic eyes [59]. These alterations may destabilise the vitreous body and by disrupting the native clearance mechanisms may go on to cause further changes which underpin the long-term increase in PPRD risk, perhaps by accelerating the process of liquefaction and syneresis that results in PVD. 
Finally, this model is further supported by studies suggesting a high incidence of PVD after phacoemulsification. A small study of 49 eyes by Ivastinovic et al. found $59.2 \%$ had new PVD at 1 month after phacoemulsification and $71.4 \%$ at 3 months [60]. A larger study of 188 eyes found $78.7 \%$ of eyes without pre-existing PVD developed one by 26 months after phacoemulsification. Interestingly, the incidence was higher $(87.23 \%)$ if the eye had pre-operative lattice degeneration (retinal thinning with separation and liquefaction of overlying vitreous) [61].

We note that Daien et al. have built on this model to suggest an explanation for the higher PPRD risk in younger eyes (in contrast to the lower phakic RRD risk in younger eyes, making age the only risk factor whose effect differs in phakic RRD and PPRD). They suggest that older eyes are more likely to have an established pre-operative PVD which is therefore protective against vitreous changes during and after phacoemulsification [11]. However, in light of Bjerrum et al.'s work using the un-operated fellow eye as a control, we would expect this to be reflected in a higher PPRD risk in younger eyes directly attributable to the procedure. In reality Bjerrum at al. found no significant difference due to age on the risk directly attributable to the procedure, instead finding the higher risk in younger eyes was entirely attributable to a higher pre-operative risk in young patients (calculated using fellow eye incidence) [38]. In fact Ivastinovic et al. found a higher incidence of new PVD after phacoemulsification in those aged over 70 (92.3\% compared with $47.8 \%$ ) [60], all of which suggests that the lower PPRD incidence in older eyes is not entirely due to the protective effect of a pre-existing PVD.

\section{Limitations}

The available literature is also limited by small population sizes. Due to the relative rarity of PPRD, even with our criteria including population $>1000$ the number of PPRDs occurring is low; only 4 of our 16 studies had more than 50 PPRDs. The available literature also frequently excluded eyes with previous RRD and some had very short follow-up durations [4]. In some studies PPRD incidence was calculated over a fixed data collection period without adjusting for the fact that the patients underwent phacoemulsification during the same time window [11, 31, 32, 39] which may lead to underestimation of the crude PPRD rate. Conversely studies with long follow-up times may overestimate the risk of PPRD attributable to phacoemulsification in younger patients due to the natural history of RRD-without adjusting for age, over long follow-up durations younger patients are more likely than older patients to have a RRD in later life which is wrongly attributed to phacoemulsification even if the durations of follow-up are equal. This may explain why Szijártó et al. found that while younger patients had a higher estimated PPRD incidence, the mean time between phacoemulsification and PPRD was longer [12].

More broadly our survey of the literature was limited by methodological differences between the different studies considered, particularly in how they quantified risk factors for PPRD, making direct comparisons more challenging. The populations of some studies are also wholly or partly subsumed in others (e.g. Olsen et al. examined phacoemulsification patients at a single Danish centre from 2000 to 2005 [35] all of whom should also be included in the Danish National Patient Registry which was used by Bjerrum et al. to examine all Danish patients from 2000 to 2010 [38]).

\section{Conclusions}

In our survey of the recent literature, PPRD remains a rare but important adverse outcome occurring in 0.36-2.9\% of cases within 10 years of phacoemulsification. In line with more recent studies, phacoemulsification in centres familiar with the technique has a similar or lower [62] PPRD incidence than ECCE. Our survey found an initially high PPRD rate which drops to about $0.1-0.2 \%$ per year for several years, still approximately ten times greater than the RRD risk of the general population.

Several patient, eye and operative factors are associated with increased PPRD risk, namely (in order of decreasing effect) intraoperative vitreous loss, increasing axial length, younger age, male sex and trainee operating surgeon. These are all the known risk factors for PPRD known from older cataract extraction methods such as ECCE, with the exception of Nd:YAG laser capsulotomy which appears not to pose a significant PPRD risk in the phacoemulsification setting and trainee operating surgeon grade which does not seem to have been investigated in the ECCE setting. Increasing age is protective, with an approximate halving of PPRD risk for each decade above 50 years. Intraoperative complications and PCR with vitreous loss in particular increase the PPRD risk more markedly in the initial post-operative phase and form the single biggest risk factor for PPRD. These risks are likely to be multiplicative to existing risks meaning that for example in a young myopic male with a previous fellow eye RRD the risk in the operative eye can be higher than perhaps previously expected.

Long-term complications of phacoemulsification such as PPRD can easily escape the notice of cataract surgeons due to increasing sub-specialisation [63]. As such, closer monitoring for RRD postoperatively with prophylactic measures e.g. retinal tear photocoagulation may have a role in identifying and preventing RRD [64]. 
While we are getting a clearer picture of PPRD incidence, the exact risk of PPRD attributable to phacoemulsification remains elusive. For more effective counselling of patients who might be at higher risk, ideally a PPRD risk calculator is needed, based on data which accounts for the limitations of the currently available literature as outlined above.

\section{Compliance with ethical standards}

Conflict of interest The authors declare that they have no conflict of interest.

Publisher's note Springer Nature remains neutral with regard to jurisdictional claims in published maps and institutional affiliations.

\section{References}

1. Hajari JN, Bjerrum SS, Christensen U, Kiilgaard JF, Bek T, La Cour M. A nationwide study on the incidence of rhegmatogenous retinal detachment in denmark, with emphasis on the risk of the fellow eye. Retina. 2014;34:1658-65.

2. Mitry D, Charteris DG, Yorston D, Rehman Siddiqui MA, Campbell $\mathrm{H}$, Murphy AL, et al. The epidemiology and socioeconomic associations of retinal detachment in Scotland: a two-year prospective population-based study. Investig Ophthalmol Vis Sci. 2010;51:4963-8.

3. Poulsen CD, Peto T, Grauslund J, Green A. Epidemiologic characteristics of retinal detachment surgery at a specialized unit in Denmark. Acta Ophthalmol. 2016;94:548-55.

4. Day AC, Donachie PHJ, Sparrow JM, Johnston RL. The Royal College of Ophthalmologists' National Ophthalmology Database study of cataract surgery: report 1 , visual outcomes and complications. Eye. 2015;29:552-60.

5. Tuft SJ, Gore DM, Bunce C, Sullivan PM, Minassian DC. Outcomes of pseudophakic retinal detachment. Acta Ophthalmol. 2012;90:639-44.

6. Javitt JC, Vitale S, Canner JK, Krakauer H, McBean AM, Sommer A. National outcomes of cataract extraction I: Retinal detachment after inpatient surgery. Ophthalmology. 1991;98:895-902.

7. Courtney P. The national cataract surgery survey: I. method and descriptive features. Eye. 1992;6:487-92.

8. Desai P, Reidy A, Minassian DC. Profile of patients presenting for cataract surgery in the UK: national data collection. Br J Ophthalmol. 1999;83:893-6.

9. Clark A, Morlet N, Ng JQ, Preen DB, Semmens JB. Whole population trends in complications of cataract surgery over 22 years in Western Australia. Ophthalmology. 2011;118:1055-61.

10. Sheu SJ, Ger LP, Ho WL. Late increased risk of retinal detachment after cataract extraction. Am J Ophthalmol. 2010;149:113-9.e1.

11. Daien V, Lepape A, Heve D, Carriere I, Villain M. Risks factors of retinal detachment following cataract surgery in a national population study between 2009 and 2012. Investig Ophthalmol Vis Sci. 2015;56:672.

12. Szijártó Z, Schvoller M, Poto L, Kuhn F, Kovacs B. Pseudophakic retinal detachment after phacoemulsification. Ann Ophthalmol. 2007:39:134-9.

13. Alio JL, Ruiz-Moreno JM, Shabayek MH, Lugo FL, Abd El Rahman AM. The risk of retinal detachment in high myopia after small incision coaxial phacoemulsification. Am J Ophthalmol. 2007;144:93-8.e2.
14. Sasaki K, Ideta H, Yonemoto J, Tanaka S, Hirose A, Oka C. Epidemiologic characteristics of rhegmatogenous retinal detachment in Kumamoto, Japan. Graefes Arch Clin Exp Ophthalmol. 1995;233:772-6.

15. Mitry DCD, Yorston D, Siddiqui MA, Campbell H, Murphy AL, Fleck BW, et al. Scottish RD Study Group. The epidemiology and socioeconomic associations of retinal detachment in Scotland: a two-year prospective population-based study. Investig Ophthalmol Vis Sci. 2010;51:4963-8.

16. Av-Shalom A, Berson D, Gombos GM, Michaelson IC, Zauberman H. Some comments on the incidence of idiopathic retinal detachment among africans. Am J Ophthalmol. 1967;64:384-6.

17. Chandra A, Banerjee P, Davis D, Charteris D. Ethnic variation in rhegmatogenous retinal detachments. Eye. 2015;29:803-7.

18. Rosman M, Wong TY, Ong SG, Ang CL. Retinal detachment in Chinese, Malay and Indian residents in Singapore: a comparative study on risk factors, clinical presentation and surgical outcomes. Int Ophthalmol. 2001;24:101-6.

19. Mowatt LS-SG, Price N. Ethnic differences in the demand incidence of retinal detachments in two districts in the West Midlands. Eye. 2003;17:63-70.

20. Wong TT, JM; Schein OD. Racial difference in the incidence of retinal detachment in Singapore. Epidemiol Biostat. 1999;117: 378-83.

21. Ghazi NG, Green WR. Pathology and pathogenesis of retinal detachment. Eye. 2002;16:411.

22. Steel DH. Retinal detachment. BMJ Clin Evid. 2014;2014. https://www.ncbi.nlm.nih.gov/pmc/articles/PMC3940167/

23. Grzybowski AKP. Does Nd:YAG capsulotomy increase the risk of retinal detachment? Asia Pac J Ophthalmol. 2018;7:339-44.

24. Michels RG, Wilkinson CP, Rice TA. Michels retinal detachment. St. Louis: Mosby; 1997.

25. Kuhn F, Aylward B. Rhegmatogenous retinal detachment: a reappraisal of its pathophysiology and treatment. Ophthalmic Res. 2014;51:15-31.

26. Tanner V, Harle D, Tan J, Foote B, Williamson TH, Chignell AH. Acute posterior vitreous detachment: the predictive value of vitreous pigment and symptomatology. $\mathrm{Br} \mathrm{J}$ Ophthalmol. 2000;84:1264-8.

27. Richardson PS, Benson MT, Kirkby GR. The posterior vitreous detachment clinic: do new retinal breaks develop in the six weeks following an isolated symptomatic posterior vitreous detachment? Eye. 1999;13:237-40.

28. Sheu SJ, Ger LP, Chen JF. Risk factors for retinal detachment after cataract surgery in Southern Taiwan. J Chin Med Assoc. 2005;68:321-6.

29. Sheu SJ, Ger LP, Chen JF. Axial myopia is an extremely significant risk factor for young-aged pseudophakic retinal detachment in Taiwan. Retina. 2006;26:322-7.

30. Sheu SJ, Ger LP, Chen JF. Male sex as a risk factor for pseudophakic retinal detachment after cataract extraction in Taiwanese adults. Ophthalmology. 2007;114:1898-903.e1.

31. Lin JY, Ho WL, Ger LP, Sheu SJ. Analysis of factors correlated with the development of pseudophakic retinal detachment-a long-term study in a single medical center. Graefes Arch Clin Exp Ophthalmol. 2013;251:459-65.

32. Quek DT, Lee SY, Htoon HM, Ang CL. Pseudophakic rhegmatogenous retinal detachment in a large Asian tertiary eye centre: a cohort study. Clin Exp Ophthalmol. 2012;40:e1-7.

33. Russell M, Gaskin B, Russell D, Polkinghorne PJ. Pseudophakic retinal detachment after phacoemulsification cataract surgery: ten-year retrospective review. J Cataract Refract Surg. 2006;32:442-5.

34. Boberg-Ans G, Villumsen J, Henning V. Retinal detachment after phacoemulsification cataract extraction. J Cataract Refract Surg. 2003;29:1333-8. 
35. Olsen $T$, Jeppesen $P$. The incidence of retinal detachment after cataract surgery. Open Ophthalmology. Open Ophthalmol J. 2012;6:79-82.

36. Hermann MM, Kirchhof B, Fauser S. Temporal occurrence of retinal detachments after cataract surgery. Acta Ophthalmol. 2012;90:e594-e6.

37. Boberg-ans G, Henning V, Villumsen J, La Cour M. Longterm incidence of rhegmatogenous retinal detachment and survival in a defined population undergoing standardized phacoemulsification surgery. Acta Ophthalmol Scand. 2006;84:613-8.

38. Bjerrum SoS, Mikkelsen KL, La Cour M. Risk of pseudophakic retinal detachment in 202226 patients using the fellow nonoperated eye as reference. Ophthalmology. 2013;120:2573-9.

39. Day AC, Donachie PHJ, Sparrow JM, Johnston RL. United Kingdom National Ophthalmology Database Study of cataract surgery: report 3: pseudophakic retinal detachment. Ophthalmology. 2016;123:1711-5.

40. Petousis V, Sallam AA, Haynes RJ, Patel CK, Tyagi AK, Kirkpatrick JN, et al. Risk factors for retinal detachment following cataract surgery: the impact of posterior capsular rupture. $\mathrm{Br} \mathbf{J}$ Ophthalmol. 2016;100:1461-5.

41. Narendran N, Jaycock P, Johnston RL, Taylor H, Adams M, Tole DM, et al. The Cataract National Dataset electronic multicentre audit of 55,567 operations: risk stratification for posterior capsule rupture and vitreous loss. Eye. 2008;23:31.

42. Ambler JS, Constable IJ. Retinal detachment following Nd: YAG capsulotomy. Aust NZ J Ophthalmol. 1988;16:337-41.

43. Tielsch JM, Legro MW, Cassard SD, Schein OD, Javitt JC, Singer $\mathrm{AE}$, et al. Risk factors for retinal detachment after cataract surgery: a population-based case-control study. Ophthalmology. 1996;103:1537-45.

44. Tuft SJ, Minassian D, Sullivan P. Risk factors for retinal detachment after cataract surgery. a case-control study. Ophthalmology. 2006;113:650-6.

45. Olsen G, Olson RJ. Update on a long-term, prospective study of capsulotomy and retinal detachment rates after cataract surgery. J Cataract Refract Surg. 2000;26:1017-21.

46. Forsell S, Monestam E. Frequency of retinal redetachment after cataract surgery in eyes with previous scleral buckling surgery. Ophthalmol Retina. 2018;2:4-9.

47. Johnston PB. Traumatic retinal detachment. Br J Ophthalmol. 1991;75:18-21.

48. Rowe JA, Erie JC, Baratz KH, Hodge DO, Gray DT, Butterfield L, et al. Retinal detachment in Olmsted County, Minnesota, 1976 through 1995. Ophthalmology. 1999;106:154-9.

49. Olsen T, Arnarsson A, Sasaki H, Sasaki K, Jonasson F. On the ocular refractive components: the Reykjavik Eye Study. Acta Ophthalmol Scand. 2007;85:361-6.
50. Mitry D, Tuft S, McLeod D, Charteris DG. Laterality and gender imbalances in retinal detachment. Graefes Arch Clin Exp Ophthalmol. 2011;249:1109-10.

51. The Eye Diseases Prevalence Research G. The prevalence of refractive errors among adults in the united states, western europe, and australia. Arch Ophthalmol. 2004;122:495-505.

52. Wang J, McLeod D, Henson DB, Bishop PN. Age-Dependent Changes in the Basal Retinovitreous Adhesion. Investig Ophthalmol Vis Sci. 2003;44:1793-800.

53. Ninn-Pedersen K, Bauer B. Cataract patients in a defined swedish population, 1986 to 1990: V. postoperative retinal detachments. Arch Ophthalmol. 1996;114:382-6.

54. Shah V, Hall N, Goldacre MJ. Retinal detachment in England: database studies of trends over time and geographical variation. $\mathrm{Br}$ J Ophthalmol. 2015;99:639-43.

55. Sharma MC, Chan P, Kim RU, Benson WE. Rhegmatogenous retinal detachment in the fellow phakic eyes of patients with pseudophakic rhegmatogenous retinal detachment. Retina. 2003;23:37-40.

56. Mahroo OA, Dybowski R, Wong R, Williamson TH. Characteristics of rhegmatogenous retinal detachment in pseudophakic and phakic eyes. Eye. 2012;26:1114-21.

57. Coppe AM, Lapucci G. Posterior vitreous detachment and retinal detachment following cataract extraction. Curr Opin Ophthalmol. 2008;19:239-42.

58. Lois N, Wong D. Pseudophakic retinal detachment. Surv Ophthalmol. 2003;48:467-87.

59. Neal RE, Bettelheim FA, Lin C, Winn KC, Garland DL, Zingler JS,Jr. et al. Alterations in human vitreous humour following cataract extraction. Exp Eye Res. 2005;80:337-47.

60. Ivastinovic D, Schwab C, Borkenstein A, Lackner EM, Wedrich A, Velikay-Parel M. Evolution of early changes at the vitreoretinal interface after cataract surgery determined by optical coherence tomography and ultrasonography. Am J Ophthalmol. 2012;153: 705-9.

61. Ripandelli G, Coppe AM, Parisi V, Olzi D, Scassa C, Chiaravalloti $\mathrm{A}$, et al. Posterior vitreous detachment and retinal detachment after cataract surgery. Ophthalmology. 2007;114:692-7.

62. Daien V, Le Pape A, Heve D, Carriere I, Villain M. Incidence, risk factors, and impact of age on retinal detachment after cataract surgery in France a national population study. Ophthalmology. 2015;122:2179-85.

63. Ducournau DH, Le Rouic JF. Is pseudophakic retinal detachment a thing of the past in the phacoemulsification era? Ophthalmology. 2004;111:1069-70.

64. Fan DSP, Lam DSC, Li KKW. Retinal complications after cataract extraction in patients with high myopia. Ophthalmology. 1999;106:688-91. 